\title{
Sobre la autoría en publicaciones académicas
}

Raúl H Sánchez-López

Editor de AUDITIO
Las consideraciones éticas y políticas editoriales de una revista académica proporcionan herramientas para asegurar el rigor y calidad de los trabajos publicados. Una de las cuestiones que a menudo se discuten es la autoría de los artículos científicos.

De forma general, se consideran autores a las personas que hayan contribuido intelectualmente y de forma sustancial a la elaboración de un estudio. Instituciones como el International Committee of Medical Journal Editors (ICMJE) recomiendan basar la definición de autor en cuatro sencillos criterios. Éstos son, y cito textualmente:

1. Que exista una contribución sustancial a la concepción o diseño del artículo o a la adquisición, análisis o interpretación de los datos.

2. Que se haya participado en el diseño del trabajo de investigación o en la revisión crítica de su contenido intelectual.

3. Que se haya intervenido en la aprobación de la versión final que vaya a ser publicado.
4. Que se tenga capacidad de responder de todos los aspectos del artículo de cara a asegurar que las cuestiones relacionadas con la exactitud o integridad de cualquier parte del trabajo están adecuadamente investigadas y resueltas.

Si un contribuidor del estudio cumple el primer criterio, se le ha de ofrecer siempre la co-autoría del manuscrito a postular, debiendo el co-autor aceptar o rechazar dicha invitación. En caso de aceptar, el co-autor deberá involucrarse de manera activa en la elaboración de artículo, ya que, si falla en cumplir los otros tres criterios, no debería ser considerado autor del manuscrito según ICMJE.

Es importante que los contribuidores acuerden de forma previa al envío, o incluso previa a la redacción del manuscrito, el papel que cada contribuidor tiene en el estudio. Puede haber contribuidores que no quieran formar parte del grupo de autores y también puede darse el caso de personas que han sido omitidas deliberadamente. En todo caso, es importante que el responsable de la investigación, o el autor encargado de la correspondencia, clarifique estos puntos antes del envío. 
Existen casos en los que algunas personas que no cumplen ninguno de los criterios de ICMJE, O únicamente uno, acaban formando parte de la lista de autores. Esto se conoce como "autoría regalada". Cuando un jefe de servicio o de departamento aparece como autor por el mero hecho de ser el responsable de la institución, pero no ha contribuido activa e intelectualmente en el desarrollo del artículo se considera "autoría regalada". En otras ocasiones se incluyen estos nombres para maximizar la posibilidad de aceptación del manuscrito, incluso sin el conocimiento de la persona implicada. En todos estos casos AUDITIO se muestra en contra de estas prácticas como viene señalado en nuestras políticas editoriales.

Otro caso es el de la "autoría fantasma", que sucede cuando un autor no ha sido incluido en un manuscrito cumpliendo los criterios de autoría. La mejor manera de atajar estos casos es llegar a un entendimiento entre los contribuidores del estudio, idealmente por escrito, acordar quienes son los autores y quienes aparecerán en los agradecimientos.

Una manera de evitar "autoría regalada" o "autoría fantasma" es el uso de Contributors Roles Taxonomy (CRediT). Los autores han de expresar en el manuscrito cuales fue el papel de cada autor utilizando 14 posibles roles, desde conceptualización hasta la revisión y edición del manuscrito final, pasando por el análisis de datos, visualización de resultados, etc. De esta manera, la revista científica tiene posibilidad de identificar casos como los anteriormente señalados más fácilmente.

En cualquier caso, disputas sobre autorías o contribuciones no pueden ser resueltas por el journal, el cuál ha de dirigirse a las instituciones a las que pertenecen los autores si éstos no llegan a un acuerdo. Si existen alegaciones sobre la autoría, el journal sigue unas pautas claras. El equipo editorial de AUDITIO, sigue las recomendaciones del comité de ética en publicaciones (COPE) y busca consejo en los casos reportados en el COPE fórum para resolver posibles irregularidades.

\section{Referencias}

International Committee of Medical Journal Editors (2019). Recommendations for the Conduct, Reporting, Editing, and Publication of Scholarly Work in Medical Journals. Archivo Disponible en ENGLISH y SPANISH.

Council of Science Editors. Authorship and Authorship Responsibilities Archivo disponible en el Internet archive

Committee on Publication ethics (2019). What to do if you suspect ghost, guest or gift authorship.

https://doi.org/10.24318/cope.2019.2.18

CRediT - Contributor Roles Taxonomy https://casrai.org/credit/

Sanchez-Lopez. R., Sobre la autoría en publicaciones académicas, AUDITIO vol. 5(2) 37-38. https://doig.org/10.51445/sja.auditio.vol5.2021.0074

https://journal.auditio.com

Publicado: 28.07.2021

(c) el Autor. Este artículo está publicado bajo licencia Creative Commons de Atribución 4.0.la cual permite el uso, redistribuición y creación de obras derivadas sin previo permiso. https://creativecommons.org/licenses/by/4.0/deed.es CC (i) 